\title{
The Protection of Intellectual Property Rights Perspective for Service Innovation Way to Study
}

\author{
Jing-jing Pian \\ School of Economics, Shanghai University, Shanghai, China
}

\section{Email address:}

jingpian406@163.com

\section{To cite this article:}

Jing-jing Pian. The Protection of Intellectual Property Rights Perspective for Service Innovation Way to Study. Science Innovation. Vol. 5, No. 3, 2017, pp. 161-166. doi: 10.11648/j.si.20170503.17

Received: March 20, 2017; Accepted: April 17, 2017; Published: April 20, 2017

\begin{abstract}
This paper mainly discusses the relationship between the protection of intellectual property and services an exploratory study. First explain the basic principles of economics, the protection of intellectual property rights because of unpaid occupy, market failure, adverse selection, non-standard market, more in need of protection of intellectual property rights; Through services innovation SSII index analysis of the importance of the protection of intellectual property rights to ensure the innovation investment returns; Finally come to the conclusion that the service innovation of intellectual property protection is becoming more and more important.
\end{abstract}

Keywords: Intellectual Property Rights (IPR) Protection, Service, Innovation

\section{知识产权保护视角下寻求服务业创新途径的研究}

\section{偏静静}

经济学院, 上海大学, 上海, 中国

邮箱

jingpian406@163.com

摘要: 本文主要对知识产权保护与服务业之间的关系进行一种探索性研究。首先解释知识产权保护的基本经济学原理, 由于存在无偿占用、市场失灵、逆向选择不规范的市场, 更需要知识产权的保护; 通过服务业创新指数SSII指数分析知 识产权保护对保证创新投资回报的重要性; 最后得出结论，对服务业创新的知识产权保护变得越来越重要。

关键字：知识产权保护, 服务业, 创新

\section{1. 引言}

普遍的观点认为, 相比制造业和农业, 服务业较不倾 向于为了实现市场价值最大化而对创新进行保护。这个观 点来源于一个现实, 即服务提供商一般不像其他产业的企 业那样频繁地申请专利, 如在欧洲的第二次协同创新调查 中发现, 只有 $7 \%$ 的服务公司申请了专利 [1]。但是在某些 服务部门, 比如软件、信息技术、娱乐和媒体生产、数据
库和商业、金融服务等, 先进技术的发展和使用, 使得这 些服务部门逐渐意识到知识产权保护在变得越来越重要。 事实上, 知识产权、各类技术和金融服务等都已成为现代 知识经济和全球化的核心。但很少有学术文献来讨论知识 产权保护在服务部门中的不同角色, 造成了很大的疏忽。 本文是对这一领域分析研究的初步尝试, 首先, 将对知识 产权保护作经济学分析, 并分析其是否适用于服务业还是 需要做一定调整; 然后分析一些服务业部门的创新情况和 知识产权保护对保证创新投资回报的重要性。 


\section{2. 知识产权保护的经济学解释}

知识产权保护包含了一系列为提高市场功能的常规 政策, 包括专利、版权、商标、商业秘密和大量相关措施。 已有很多理论和经验文献分析了知识产权保护的必要性, 归纳起来, 主要牵涉不规范市场中的三个关键问题:

(1) 无偿占用。一些新的发明和创意可能会被模仿, 比如, 计算机软件、药品和生物技术就较容易被模仿, 这 将阻碍发明者从中获取足够的利润, 以弥补其研发成本。 即新思想和新信息可能不是独占的, 发明者不能实现其经 济价值。而专利和版权能提供创造者排他的权利, 这种法 定的排他性能保证创新的最初发明者获得利润, 为他们的 投资提供了外部动机。

（2）市场失灵。在将新技术引入市场的过程中，会 产生很多交易成本, 其中有两个特别重要。第一种重要的 交易成本发生于技术许可过程中，投资者希望授予其他公 司许可权，但这样做就会泄露他们的技术和方法。虽然许 可证持有者在他们完全理解技术的工作原理和明确执行 成本之前不会违反合同, 但创新者也需要有足够信心保证 信息不会被泄露。此时专利、版权、商业秘密和商标等都 是支持技术许可证发放或特许合同签订的保护方法。第二 种重要的交易成本形式是发明者和被许可方需要对市场 渠道、广告和互补性服务进行投资, 这些是成本型投资, 而且只有在保证排他性情况下才会产生。

（3）逆向选择。企业需要在他们的品牌上进行大量 投资，否则将面临被伪造的风险。如果没有保护，消费者 就不能保证自己购买的是高质量的产品, 也就不会再愿意 支付高价购买, 结果是高质量的产品被完全挤出市场, 这 是不完全市场中典型的逆向选择。此时, 商标是对信息问 题的一种很好的解决方法。

总之, 知识产权保护扮演了一个双重角色。一方面, 作为创新的动机来源; 另一方面, 作为一项法律法规以减 少交易成本。但创新不仅会被占用，同时还会产生消极的 不竞争, 社会希望这些商品分布尽可能广, 而价格尽可能 低, 但这是与强有力的专利分配相冲突的, 这也就是知识 产权保护中存在的普遍问题和困难。尤其是专利, 会产生 被保护市场势力和滥用的许可行为。比如, 微软占据了操 作系统的控制地位, 并扩大了对应用程序的市场势力。但 这个问题在完全竞争和有秩序的市场中不可能发生, 因为 存在相互竞争的技术和产品, 比如微软的市场垄断会鼓励 竞争软件产品的进入。另一个问题是, 创新可能是创造价 值的过程, 每一步都是建立在前面的知识基础之上。如果 专利和商业秘密非常强, 这种创造价值的创新将会被阻碍 [3]。

\section{3. 服务业创新和知识产权保护的作用}

虽然多数服务行业是知识产权产品和技术的使用者, 但某些服务行业也是创新思想的生产者。这一部分描述了 服务业创新的特征, 这里要解决的问题是服务部门在多大 程度上符合知识产权保护的基本经济框架。

\section{1. 服务业创新的特征和IPR的相关性}

服务业是一组大量不同性质的经济和社会活动的集 合。按要素密集度分类, 服务业包括 (1) 资本密集型服 务。这类服务包括空运、通信、工程建设服务等。（2） 技术与知识密集型服务。这类服务包括银行、金融、法律、 会计、审计、信息服务等。（3）劳动密集型服务。这类 服务包括旅游、交通运输、建筑、维修、消费服务等。这 种明显的性质差异意味着很难制定一个统一的知识产权 保护标准, 能平等地适用于所有部门。事实上, 只有一部 分服务种类符合知识产权保护的经济学模型, 这部分服务 类别具有高昂的固定投资成本、低 (或零)边际生产成本、 无竞争以及部分无排他性消费等特征。服务业中符合这种 描述的有软件、录制娱乐产品、电视和广播、研发服务和 数据库等, 这些行业能提供大量的知识产权, 且通常涉及 大量的投资成本以及巨大的被非法占用的风险。对私人公 司来说, 要控制创新的溢出会产生很大的交易成本, 从而 降低它们将创新商业化的意愿。因此, 在这些领域就存在 知识产权保护的经济学意义。

但是, 在对服务业创新的知识产权保护上存在很多争 议。其中, 一个较受争议的创新种类是商业方法, 如组织 金融交易的编码方法（通常是一种计算机程序）、计算方 法、库存控制程序和其他类似的计算编码方法等。这些技 术的创新和使用对服务业非常重要, 是整个产业在生产过 程中的核心投入, 尤其在技术与知识密集型服务业, 发明 这些创新会产生直接的投资成本以及被模仿的风险, 这增 添了创新公司对创新保护的需求。由此, 美国赋予了商业 方法创新的专利保护法律资格, 但对于这些商业方法是否 确实具有创新性存在很大争议。EU目前更倾向于保护商业 秘密, 但是对商业方法要求专利化的压力也在越来越大 [4]。

除此之外, 对其他服务创新种类也存在较大的争议。 这些服务产品可能包含在一些知识产权产品的某些特性 中。比如银行服务, 发明一种存款需求通常不会产生巨大 的投资成本, 但是这种创新的模仿成本很低, 使得这些存 款很可能成为纯粹的竞争性产品, 导致发明银行无法实现 其创新的收益, 法律服务也是如此。在运输, 通讯, 健康 和教育服务业中，虽然对固定资产的投资远远高于对发明 技术的投入，但在这些服务业中，更重要的是对人力资本 的投资, 不过在入职之前这种投资大部分是由个体承担的, 投资获取的技能主要是非竞争性的, 同时也是具有个体差 异的。因此, 鼓励这种创新投资的IPR保护的范围就非常 小。这种争论在零售和批发经销以及劳动密集型服务业中 尤其激烈。

所以，除了那些知识产权密集型服务行业之外，几乎 没有服务部门需要专利或版权来保护其创新。这些服务部 门中的创新主要包括机构的组织变革、生产线以及商店零 售空间的扩大、金融衍生品的引入等, 其中没有一个适用 于正规的IPR保护。

此外, 还存在其他一些原因导致IPR保护在很多服务 创新中的作用有限。首先, 服务创新过程的产出通常是无 形的, 不能被包含在有形体中, 而通常是包含在技术工人 
的技能中。事实上, 人力资本对服务业中的非正式创新, 比正式创新和制造业中的R\&D创新更关键。

其次, 在很多服务业中, 新的知识是由服务提供商和 客户之间的同步互动产生的。在高技术服务部门, 如工程、 科学研究、技术和商业咨询、软件和计算服务以及公司财 务, 服务的实现通常由提供商和顾客共同完成。客户经常 是创新所需信息的来源, 并且能独自实现后续发展。因此, 要明确一项IPR的发明者到底是谁, 或者怎样分配可能并 不十分清晰。

再者, 绝大多数的服务生产商是中小规模的企业, 相 对于大企业来说, 它们可能更少开展正式的创新活动。因 此, 小企业几乎不申请正式的IPR保护。这种结果是一方 面, 新设立的服务公司比大企业更难融资, 因为它们不能 提供专利作为担保; 另一方面, 要使一项新技术或新的服 务产品在被商业化之前显现其市场价值可能也比较困难。

最后，除了高技术服务业之外，大部分服务企业没有 单独的R\&D部门, 而是由其他一些研发服务公司来充当, 例如特效公司相对于电影制造商, 大学实验室相对于医药 和生物公司来讲, 都可被视为后者的R\&D部门。除此之外, 金融、工程和计算服务公司也可能具备专业的R\&D功能。 虽然这些专门的R\&D实体在不断地为它们的研发产出（如 公式、基因序列、软件和数据库等) 登记专利和版权, 但 由于缺乏独立的R\&D部门, 这些服务活动本身并没能被实 行保护。

表1 2011年WIP0服务业商标注册分类、分国家比较。

\begin{tabular}{|c|c|c|c|c|}
\hline Class Origin & China & Canada & USA & Japan \\
\hline 35 & 28239 & 883 & 28107 & 1354 \\
\hline 36 & 9392 & 358 & 12953 & 556 \\
\hline 37 & 11472 & 297 & 5361 & 844 \\
\hline 38 & 5363 & 227 & 5032 & 504 \\
\hline 39 & 8739 & 195 & 3520 & 384 \\
\hline 40 & 5941 & 225 & 2983 & 491 \\
\hline 41 & 16068 & 633 & 24913 & 1414 \\
\hline 42 & 13377 & 720 & 15333 & 999 \\
\hline 43 & 23434 & 123 & 6034 & 524 \\
\hline 44 & 10093 & 132 & 6253 & 377 \\
\hline 45 & 2393 & 199 & 4729 & 291 \\
\hline 服务类商标注册比重 & 17. $39 \%$ & $37.80 \%$ & 43. $33 \%$ & $19.52 \%$ \\
\hline
\end{tabular}

数据来源: http://www. wipo. int/ipstats/en/statistics/marks/ 注: 根据尼斯分类第九版, 商标注册类别中 35-45类属于服务业领域

所以，专利和版权在服务产业并不普及。相反，大部 分服务企业则依赖于商标保护 (包括商品商标、服务商标 和集体商标) 来解决信息问题以及向消费者发送质量信号。 与制造业企业相同, 商标对于银行、航空、酒店连锁和饭 店等服务行业同样非常重要, 因为商标可以使这些服务提 供商建立质量信誉, 而不用担心搭便车者会通过伪造自己 的品牌和商标而破坏它们的形象。表1是2011年中国、加 拿大、美国和日本四个国家在WIP0中的服务类商标注册数 据。从服务类商标注册比重来看, 服务业商标注册在商标 注册总量中具有一定的分量, 特别是美国和加拿大的较大 比重, 就很好地说明了商标在服务业创新保护中的重要性。 从数量上看, 中国服务业创新商标注册量几乎都超过这些
发达国家, 但是占总体商标注册量的比例却都低于它们。 说明虽然我国服务企业的商标注册绝对量非常大, 而且呈 逐年上升趋势（见图1），但与其他产业相比，仍然存在 一定的差距。

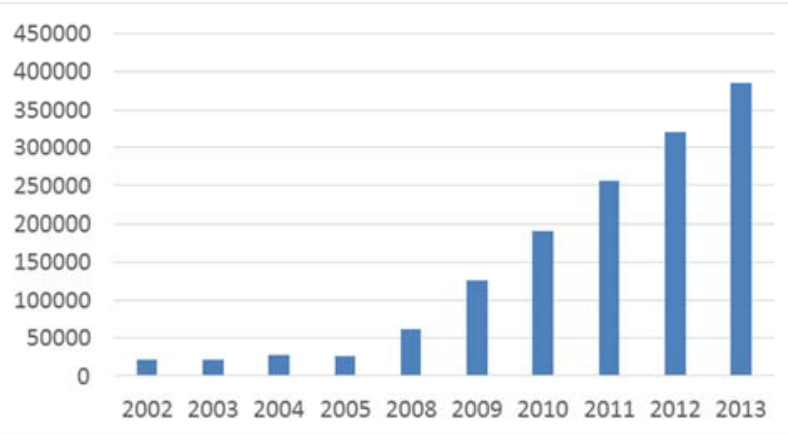

数据来源: 2002-2013年《中国商标战略年度发展报告》

图1 我国2002-2013服务类商标注册量。

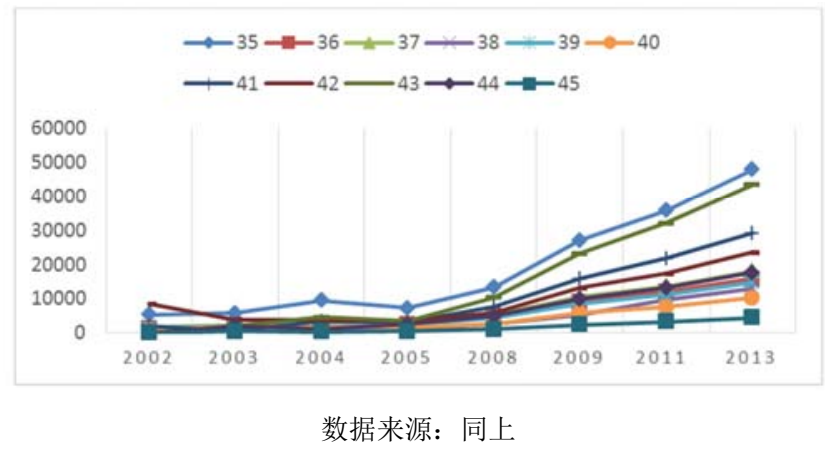

图2 我国2002-2013服务类商标注册量分类比较。

从商标种类上看（见图2），2002-2013年期间我国服 务类商标注册中, 第35、43、41类服务业的商标注册量较 大、增长速度也较快, 它们分别是广告、实业经营和管理, 食物与饮料服务、临时住宿和教育培训、娱乐文体活动, 而知识密集型服务行业 42 类, 即科学技术服务和与之相关 的研究与设计服务、工业分析与研究、计算机硬件与软件 的设计与开发等在商标注册数量上并不明显, 而这类服务 在发达国家的商标注册中非常活跃。说明我国服务业的发 展仍然集中于劳动和资本密集型服务行业, 在高技术和知 识密集型的服务业上与发达国家仍有差距。

综上所述, 有一部分服务产品充分带有知识产权商品 的特性，依赖于专利和版权保护。而更多的服务企业拥有 的则是大量的商业秘密, 比如客户名单、逻辑运算方法和 配方等。而且大部分服务产品依赖商标保护以传递质量信 号。显然, 这些不同的子部门在创新种类和创新保护方法 上存在很大的差异。

\section{2. 衡量和指标}

由于服务业创新的复杂性, 要建立一个一致性、可比 较的服务创新衡量方法比较困难。简单地使用R\&D支出来 代表创新的产出是存在缺陷的, 即使一个公司一年的R\&D 支出有所下降，这也并不必然说明创新的减少，因为企业 在创新过程中, 可能是通过雇佣技术工人, 而不是通过正 
规的R\&D投入形式来实现的。而且服务创新的产出通常是 无形的, 不适用于如专利等保护方法。因此, 各部门和各 国家之间的比较就存在问题。

由于这些原因, 这一部分仅对现有的衡量服务创新的 方法做一个简单的回顾，以帮助汲取其中的一些实验性经 验。

\section{2. 1. R\&D支出}

Hane1 (2004) 指出, R\&D统计数据对各类服务产业中的 研究与开发活动的描述是不完全和扭曲的。但如果我们暂 时不考虑这一缺陷, 服务业中的R\&D数据能使我们得到一 些有意义的发现。表2考察了美国和加拿大的工业部门和 主要服务业部门的R\&D投入强度（R\&D支出/总收入）。由 于两个国家之间的具体情况有所不同, 比如美国医院相比 于加拿大医院更依赖于进行大量内部研发的大学, 所以美 国卫生保健部门的这一比率比加拿大低很多。因此, 国家 内部的比较在一定程度上更加合理。在美国和加拿大, 服 务行业的R\&D投入强度几乎与制造业同样重要。尤其是一 些高技术服务部门 (软件业、科学技术服务业、工程、计 算机服务、科技R\&D部门、卫生服务、管理咨询）的R\&D 投入强度甚至高于研发密集型的制造业。

表3 列出了我国各行业在2000年、2002年和2011年的 $R \& D$ 投入强度（R\&D支出/产业增加值）。由于数据统计的 标准不同，在行业划分和数据使用上，中国各行业R\&D投 入强度的统计结果与美国和加拿大的情况有所区别, 但仍 然在一国内部比较, 可以发现我国的地质勘查和水利管理 业、交通运输和仓储业邮电通信业、卫生业、教育、文化 艺术及广播电影电视业、科学研究业、综合技术服务业及 其他等服务行业的R\&D投入强度与工业制造业相近甚至高 于。
在一个国家内部，直接观察到的是不同部门将销售收 入用于R\&D支出的比例有很大的差异。2000年，美国所有 制造业的研发与收入总体比率为 $3.6 \%$, 当然这一数字在各 个制造业行业中是存在变化的。但是在服务部门, 科学研 究服务贡献了 $43 \%$ 的总收入用于R\&D, 主要被用于支付专业 研究人员的工资。其他高技术研发部门还包括软件、计算 机系统设计以及建筑和工程服务。建筑和贸易也比其他所 有制造业部门更具有研发密集性。总的来说, 加拿大的多 部门等级排序与美国的差不多。这里主要应注意的是各类 服务部门在对新技术的投资中有很大的不同。一些部门从 事大量的研发, 如知识密集型服务业, 而这些行业在研究 领域也正在引起越来越多的关注。

表2 2000年美国和加拿大各行业R\&D投入强度比较。

\begin{tabular}{lll}
\hline 行业 & US (2000) & Canada(2000) \\
\hline 制造业 & 3.6 & 1.9 \\
建筑业 & 5.8 & 0.8 \\
贸易 & 5.4 & 1.6 \\
运输、仓储业 & na & 1.9 \\
信息部门 & 4.1 & 0.2 \\
报纸期刊、书籍出版业 & 2 & 1.5 \\
软件 & 20.5 & na \\
金融、保险、房地产 & 1.2 & 0.2 \\
专业、科学技术服务业 & 18.3 & na \\
建筑工程 & 10.8 & 15.2 \\
计算机系统设计 & 12.3 & 13 \\
科技R\&部门 & 42.9 & 32.7 \\
管理咨询 & 4.4 & 11 \\
卫生服务 & 3.2 & 35.4 \\
其他非制造业部门 & 1.1 & 1.3 \\
\hline
\end{tabular}

来源: Hane1 (2004)

表3 中国各行业R\&D投入强度。

\begin{tabular}{lll}
\hline 行业 & 2000 年 & 2002 年 \\
\hline 工业/制造业 & 0.012383025 & 0.000149407 \\
建筑业 & 0.000895649 & 0.00013409 \\
地质勘查业、水利管理业 & 0.015216068 & 0.006888733 \\
交通运输和仓储业邮电通信业 & 0.00179344 & 0.000320779 \\
计算机应用服务业 & na & na \\
批发和零售贸易餐饮业 & 0 & $6.96026 \mathrm{E}-07$ \\
金融、保险业 & 0 & $5.57246 \mathrm{E}-05$ \\
房地产业 & 0 & $3.81279 \mathrm{E}-07$ \\
社会服务业 & 0 & 0.000187683 \\
卫生业 & 0.0125157675 \\
教育、文化艺术及广播电影电视业 & 0.014526086 & 0 \\
科学研究业、综合技术服务业及其他 & 0.030779525 & 0.006176806 \\
国家机关、政党机关和社会团体 & 0.30946036 & 0.000235108 \\
\hline
\end{tabular}

数据来源：中国科技统计（http://www. sts. org. cn）和中国国家统计局网（http://www. stats. gov. cn）。 $n a ：$ 无可用数据

\subsubsection{SSII}

Kanervaet a1.（2006）的研究提出了一个衡量EU成 员国国内服务业创新的指数SSII [4]。这个服务部门创新 指数总共包含 24 个指标, 分为 7 个方面内容: 人力资源; 创新需求; 科技知识; 非技术性变革, 如组织创新; 知识 的来源和传播; 商业化; 以及知识产权的使用。因为无法
获取所有服务部门的指标数据, 这个指数只覆盖四个主要 的服务部门：（1）批发贸易;（2）交通、仓储和通信; （3）金融中介服务; (4) 计算机、R\&D和其他商业服务。

利用上述方法得到了一些有趣的结果。比如, 2005 年这 25 个EU成员国的所有制造业部门在三个类别中的成 分指数超过了服务部门的平均水平, 这三个类别是科技知 
识, 商业化和IPR的使用。这一结果也证实了服务创新比 制造业创新更具无形性的本质。从本质上来讲, 服务业的 创新更倾向于建立在公司内部的人力资本和非技术性变 革的实施上。这个研究还提供了这些国家的一个综合指数。 这个指数范围从保加利亚的 0.33 到瑞典的 0.56 。

但是, 使用SSII时必须要当心, 因为它并不与其他 $\mathrm{EU}$ 创新指数或者收入水平有很好的关联性。比如, 拉脱维亚
和捷克共和国分别排名第三和第四, 而丹麦则接近于末尾。 作者给出的解释是在服务业中要达到相同的技术水平尤 其简单，因为对无形创新的模仿相对来说更容易，而且也 很少有限制性的专利保护。考虑到这方面, SSII就无法区 别哪些企业是新工艺和新产品的发明者, 而哪些企业只是 扮演了这些新工艺和新产品的引进者。

表4 2011年EP0专利申请: 分国家和技术领域。

\begin{tabular}{|c|c|c|c|c|c|c|c|}
\hline 申请国家 技术领域 & 影音媒体 & & 计算杭 & & 电信 & & 服务业比重 \\
\hline Canada & 146 & $7.1 \%$ & 245 & $12.0 \%$ & 367 & $18.0 \%$ & $37.1 \%$ \\
\hline China & 101 & $6.2 \%$ & 65 & 4. $0 \%$ & 590 & $36.2 \%$ & $46.4 \%$ \\
\hline Japan & 1901 & $9.5 \%$ & 826 & 4. $1 \%$ & 1071 & $5.4 \%$ & 19. $1 \%$ \\
\hline United States & 1811 & $5.5 \%$ & 2494 & $7.6 \%$ & 2203 & $6.7 \%$ & 19. $7 \%$ \\
\hline
\end{tabular}

数据来源: http://www. epo. org/about-us/office/statistics/patents-technology. html

表5 我国服务业创新率（商标注册量/R\&D投入）分类比较。

\begin{tabular}{lll}
\hline 商标类别 & 2002 年 & 2011 年 \\
\hline 35 & na & na \\
36 & $38.2 \%$ & $83.1 \%$ \\
37 & na & na \\
38 & $8.8 \%$ & $0.3 \%$ \\
39 & $34.5 \%$ & $7.8 \%$ \\
40 & na & na \\
41 & $2.6 \%$ & $0.3 \%$ \\
42 & $0.3 \%$ & $0.1 \%$ \\
43 & 0 & $5.1 \%$ \\
44 & 0 & na \\
45 & 0 & na \\
\hline
\end{tabular}

数据来源: 中国科技统计网站 (http://www. sts. org. cn) 和2002、2011 年《中国商标战略年度发展报告》

\section{4. 结论}

服务业, 尤其是那些进行大量R\&D投资的高技术部门, 已逐渐成为高收入经济体的一个创新部门, 而且国际服务 贸易也在迅速增长, 这些趋势使得服务产业发生了结构转 变和持续的全球化, 这将导致就业的增长和对技术工人需 求的提高。因此, 对服务业创新的知识产权保护变得越来 越重要。

目前, 一些成熟的专利保护系统主要是鼓励制造业和 其他非服务企业的创新, 未来专利保护范围是否能扩展到 前期的服务业创新, 答案是不确定的, 这需要政策制定者 认真的思考。但一个有效的方法是努力提高服务提供商的 意识和知识, 使他们清楚专利保护的范围以及保护成本和 收益, 并进一步通过检验程序上的跨国合作来降低获取国 际保护的成本。例如通过建立欧洲共同体专利体系, 可以 降低EU区域内的专利申请成本。

至于商业方法专利的相关规定, 可能可以帮助金融公 司和电子商务等服务商保护其创新成果。但是，这种专利 或许是不妥当的, 因为它会降低创新性的标准, 并提高使
用者的交易成本；其次，没有充足的证据证明对这些技术 的专利保护能比商业秘密保护更能激励创新。

在世界水平上, 特定的服务产业会遇到不同的保护水 平, 这在软件、音乐和录像等服务产业中最为明显。这就 必须鼓励一些发展中国家重视它们的保护责任 [5]。

另外，如果知识产权制度之外的措施同样有效，那么 对服务业的自由化、减少小型服务企业进入资本市场的阻 碍, 可能也是激励更多创新的途径。

\section{参考文献}

[1] Branstetter, L., Fisman, R., and Foley, C. F., “Do stronger intellectual property rights increase international technology transfer? Empirical evidence from US Firm-Level panel data, ” Quarterly Journal of Economics, vol.121, no. 1, pp. 321-349, 2006.

[2] Fraunhofer Institute, Patents in the service industries: Final rHoekman, B. M., "Trade in services, trade agreements and economic development: A survey of literature, " Centre for Economic Policy Research, Discussion Paper, no. 5760, 2006.

[3] Jaffe, A. B., and Lerner, J., Innovation and its discontents: How our broken patent system is endangering innovation and progress, and what to do about it. Princeton: Princeton University Press, 2004 .

[4] Kanerva, M., Hollanders, H., and Arundel, A., Trendchart report: Can we measure and compare innovation in services? Maastricht Economic Research Institute on Innovation and Technology, 2006.

[5] Maskus, K. E., Reforming U.S. patent policy: Getting the incentives right, Council on Foreign Relations, Council Special Report no. 19, November, 2006. 
[6] Maskus, K. E., and Lahoue1, M., “Competition policy and intellectual property rights in developing countries, " The World Economy, vol. 23, no. 4, pp. 595-611, 2000.

[7] Mendonca, S., Pereira, T. S., and Godinho, M. M. , "Trademarks as an indicator of innovation and industrial change,” paper presented at the DRUID Summer Conference on Industrial Dynamics, Innovation and Development, Denmark, 2004.
[8] 刘晓惠, 华德亚. 促进我国服务业知识产权保护的途径研究 [J].2014（08）：104-105。

［9］李伟, 董玉鹏. 产业自主创新视角下的知识产权服务业发展 问题研究 [J].2015 (10) :142-146。

[10] 唐保庆, 张伟, 顾晓燕. 知识产权保护影响服务业发展的国 内外研究新进展 $[J]$. 2013 (10) :66-72。 